\title{
Effect of group grounding on the potential rise across solar PV panels during lightning strike
}

Faisal Peer Mohamed

Systems Engineeering Department

Military Technological College,Minisry of Defence

Sultanate of Oman,

Faisal.Mohamed@mtc.edu.om

\author{
Wah Hoon Siew \\ Dept of Electronic \& Electrical \\ Engineering, Royal College Building, \\ University of Strathclyde, Glasgow, \\ G11XW, United Kingdom \\ wh.siew@strath.ac.uk
}

\author{
Shekhar Mahmud \\ Systems Engineeering Department \\ Military Technological College,Minisry \\ of Defence \\ Sultanate of Oman, \\ Shekhar.Mahud@mtc.edu.om
}

\begin{abstract}
Grounding systems play a vital role for dissipating the energy originating from a lightning stroke to the earth, functioning as an important component for protection and safety. In a solar photovoltaic (PV) farm, solar PV panels are fixed on a grounded structure with bolts and nuts. The structure, the frame of the PV panels, and the bolts and nuts are metallic (together called the assembly) and the layout of all assemblies of the entire solar farm depends on the terrain where they are installed. Lightning protection systems which are installed on a solar PV farm are mostly based on a Franklin rod (connected to a down-conductor) as the preferred point of attachment. These lightning rods can be installed either as isolated systems or as non-isolated systems from the solar panel assemblies. This paper is focused on the effect of group grounding on the potential rise across the solar PV panels during lightning strike. This whole assembly is simulated for various lightning attachment points in PSPICE using the lossy transmission line model. Voltage drop at various points in the assembly is measured for various soil resistivity. Based on the simulation results, group grounding of solar PV panels with middle grounding shows relatively low voltage drops compared to end grounding.
\end{abstract}

Keywords- Solar PV panels, Lightning protection systems, grounding

\section{INTRODUCTION}

Higher penetration of photovoltaic system put pressure on the service providers regarding operational security and safety in particular under extreme lightning conditions like thunderstorms [1]. In a solar photovoltaic (PV) farm, solar PV panels are fixed on a grounded structure with bolts and nuts. The structure, the frame of the PV panels, and the bolts and nuts are made up of metal. Lightning protection systems which are installed on a solar PV farm are mostly based on a Franklin rod (connected to a down-conductor) as the preferred point of attachment. Consequently, it utilizes the concept of protective angle or rolling sphere method to determine the protective zone to the solar panel assemblies. Despite the installation of the lightning protection system (LPS), direct lightning strikes to the solar PV panel frame/structure might still happen. The general strategies in installing the PV system components and location design for optimized efficiency of power production should be compatible with strategies of lightning protection design [2].
Depending on the point of strike, the maximum impulse current and the soil resistance there is a possible risk that the voltage drops along the PV -module mounting system which might damage the panel(Benesova, Haller et al. 2012). Ayub et al (Ayub, Siew et al. 2018) has compared the effect of individual grounding of solar PV assemblies based on either end grounding or middle grounding on distributed voltage drop across PV panels for different types of soil. Based on his findings middle grounding does not offer any obvious advantage over end grounding for different soil resistivity [3]. This research investigates the effects of direct lightning strikes onto a solar PV assembly by considering the overvoltage resulting on the system due to various grounding arrangements. In particular, this paper is focused on group grounding of solar PV panels in which effect of middle-grounding or end-grounding points to down conductors for various soil resistivity is compared for distributed voltage drops across solar PV panels. This paper is organized into five sections. Research methodology is covered in section II. /in section III modelling parameters are discussed. Results and discussions are included in section IV. Section V concludes the paper.

\section{METHODOLOGY}

In this investigation, group grounding strategy is used to analyse the potential drop across solar PV panels during lightning strike. RLC circuit model of solar PV panel is extracted from the panel specifications and simulated in SPICE transient simulation using current source as lightning leader. Voltage drops across various points were measured in time domain and analysed using Matlab.

\section{MODELLING PARAMETERS}

\section{A. Modelling Solar PV Panel}

The whole system consists of four PV assemblies. Each PV assemblies consisting of 10 PV panels. The assembly considered consists of ten units of $350 \mathrm{~W}$ panels occupying an area of $5 \mathrm{~m} \times 4 \mathrm{~m}$ with a total output of $3.5 \mathrm{~kW}$. The dimensions of each solar frame are $1 \mathrm{~m}$ width and $2 \mathrm{~m}$ length and its cross-section is $2 \mathrm{~cm}$ width and $5 \mathrm{~cm}$ height and it is as illustrated in Figure 1. 


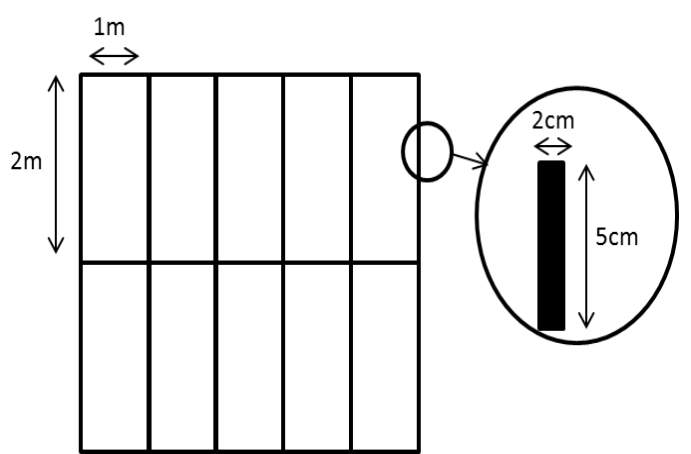

Figure 1. Illustration of an assembly considered and the blow-up image is the cross section of solar frame

The model is simulated for two scenarios namely endground and middle-ground. In the end ground, grounding is connected to one end of the four assemblies as shown in Figure 2. While in middle grounding, grounding rod is connected to mid-point of each of the four assemblies as shown in Figure 3.

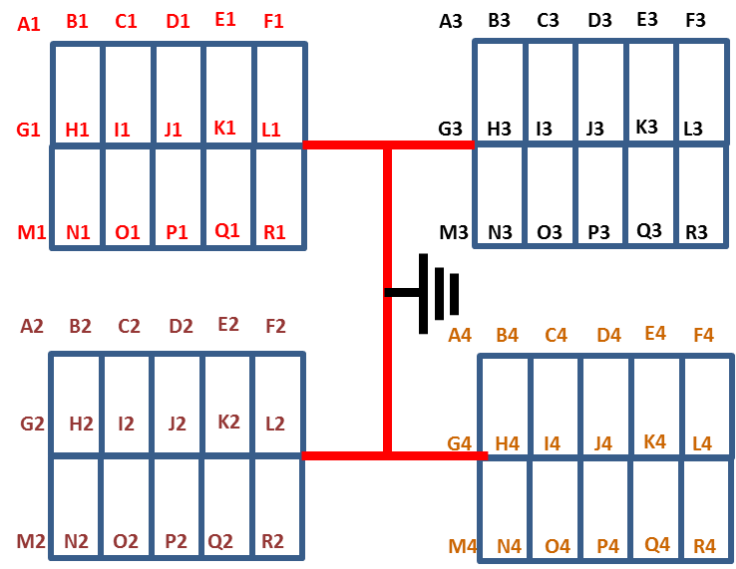

Figure 2 Solar PV assembly, end-point group grounding

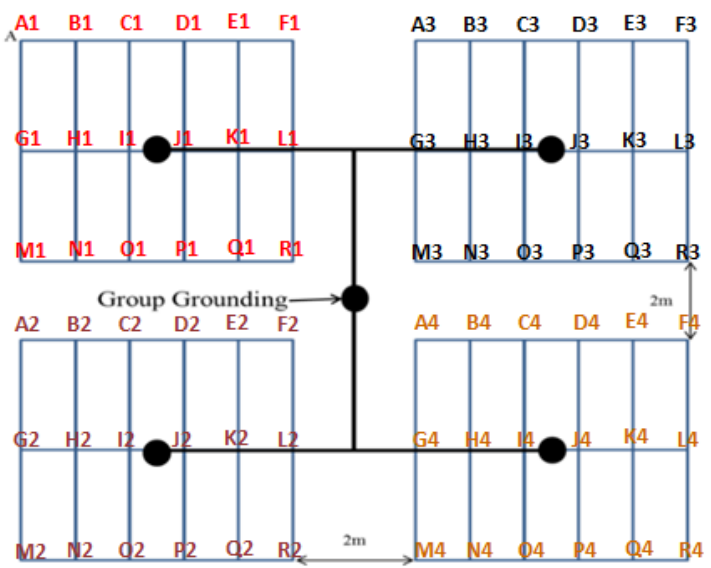

Figure 3 Solar PV assembly, middle-point group grounding

SPICE model of solar assembly with middle grounding is shown in Figure 4.

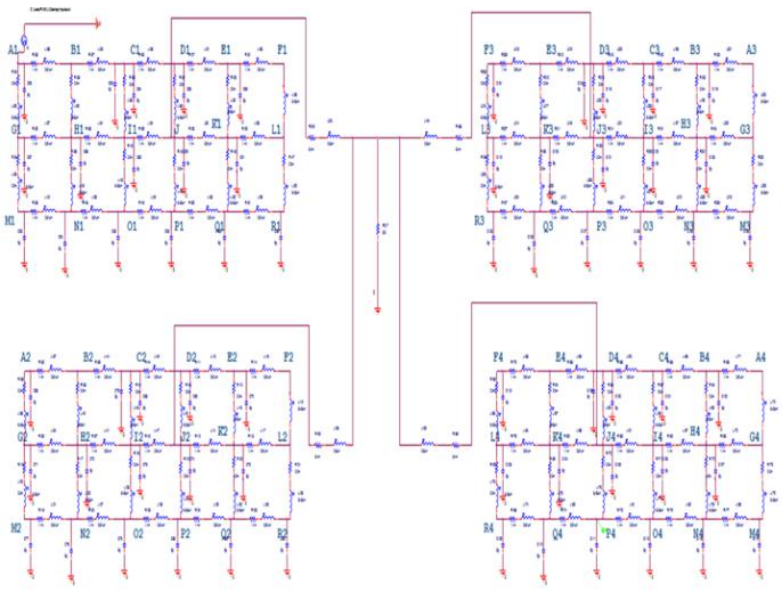

Figure 4. SPICE model consisting of four assemblies with midpoint grounding

\section{B. Lightning Impulse Current Prameters}

The lightning current considered in this investigation is defined by the double exponential expression as shown in equation 1

$$
i=\frac{I}{k} \times \frac{\left(t / \tau_{1}\right)^{10}}{1+\left(t / \tau_{1}\right)^{10}} \times e^{\left(-t / \tau_{2}\right)}
$$

where $\mathrm{I}$ is the peak current, $\mathrm{k}$ is the correction factor for the peak current, $\mathrm{t}$ is the time, $\tau 1$ is the front time constant, and $\tau 2$ is the tail time constant. Figure 5 shows the lightning current impulse waveform generated from (1) using parameters tabulated in Table 1 below.

Table 1 Parameters for Lightning Protection Level (LPL) 1 for subsequent stroke $0.25 \mu$ s (front time) $/ 100 \mu$ s (tail time)

\begin{tabular}{|c|c|}
\hline Parameters & Lightning Protection Level (LPL) 1 \\
\hline$I(\mathrm{kA})$ & 50 \\
\hline $\mathrm{k}$ & 0.993 \\
\hline$\tau_{1}(\mu \mathrm{s})$ & 0.454 \\
\hline$\tau_{2}(\mu \mathrm{s})$ & 143 \\
\hline
\end{tabular}

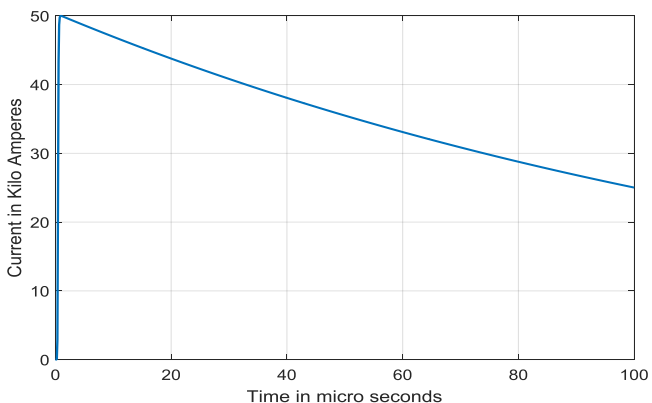

Figure 5. Lightning current impulse waveform of $0.25 \mu \mathrm{s} / 100 \mu \mathrm{s}$ (generated from (1) using parameters tabulated in Table 1)

\section{RESULTS AND DISCUSSION}

In each of the grounding strategy having different soil resistivity, an impulse current is injected at point $\mathrm{A}$ of the assembly shown in Figure 2 and Figure 3.Potential drops were measured at points A I L and P across four assemblies for various soil resistivity.

\section{A. Group Assembly - Endpoint ground}

Potential drops at locations A I L and P are as shown in Figure 6 - Figure 9.

Referring Figure 6, voltage drop at the injection point A of the solar PV system has reached $3400 \mathrm{kV}$ for soil resistivity 
of $100 \Omega$-meter which is $30 \%$ more compare to the voltage drop point $\mathrm{A}$ of the same PV system installed in a soil resistivity of $10 \Omega$-meter. Voltage drop at point $\mathrm{I}$ is $2700 \mathrm{kV}$ for $100 \Omega$-meter which is $50 \%$ more than that of $10 \Omega$-meter. As per the Figure 7 , voltage drop at the injection point $L$ of the solar PV system has reached $2700 \mathrm{kV}$ for soil resistivity of $100 \Omega$-meter which is $90 \%$ more compare to the voltage drop point $\mathrm{A}$ of the same PV system installed in a soil resistivity of $10 \Omega$-meter. Voltage drop at point $\mathrm{P}$ is $2655 \mathrm{kV}$ for $100 \Omega$-meter which is $50 \%$ more than that of $10 \Omega$-meter. Referring to Figure 8 Figure 9 Figure 14, Figure 15, Figure 16 and Figure 17, potential distribution in rest of the other assemblies (assemblies 2,3 and 4) are similar. Maximum voltage drop attains $2655 \mathrm{kV}$ for $100 \Omega$-meter which is $90 \%$ greater than the solar PV system installed in a soil having resistivity of $10 \Omega$-meter.

Voltage drop in the assembly in which lightning strikes, it is found that potential drops gradually decreases as approaching the ground point. It is found that in rest of the PV assemblies' potential drops across the points (A I L and P) are similar.

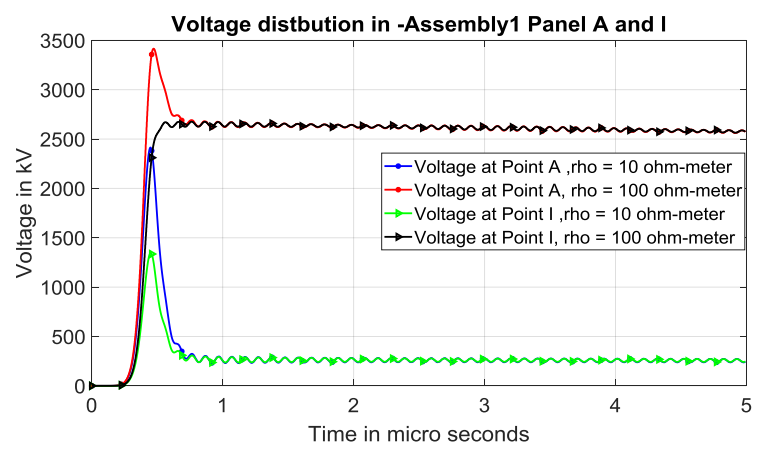

Figure 6 Potential drops in Assembly 1 - end-grounding for soil resistivity of $10 \Omega-\mathrm{m}$ and $100 \Omega-\mathrm{m}$

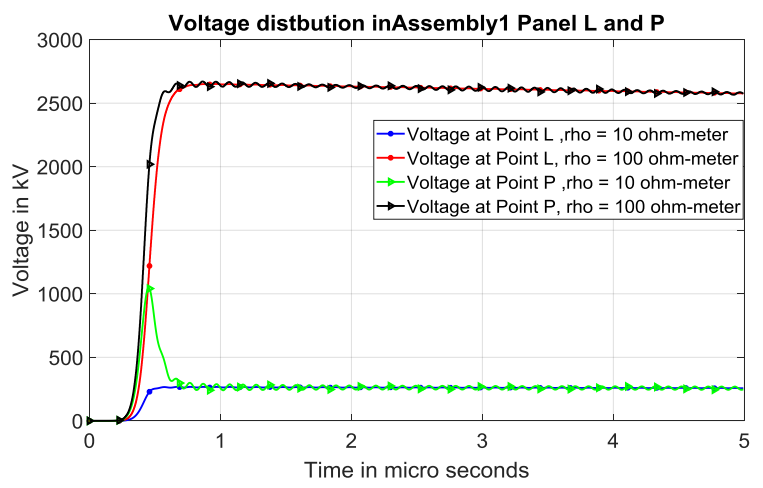

Figure 7 Potential drops in Assembly 1 - end-grounding for soil resistivity of $10 \Omega-\mathrm{m}$ and $100 \Omega-\mathrm{m}$

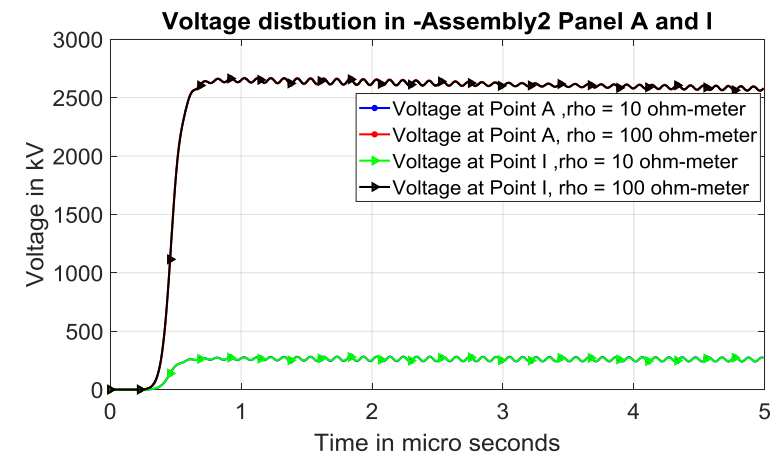

Figure 8 Potential drops in Assembly 2 - end-grounding for soil resistivity of $10 \Omega-\mathrm{m}$ and $100 \Omega-\mathrm{m}$

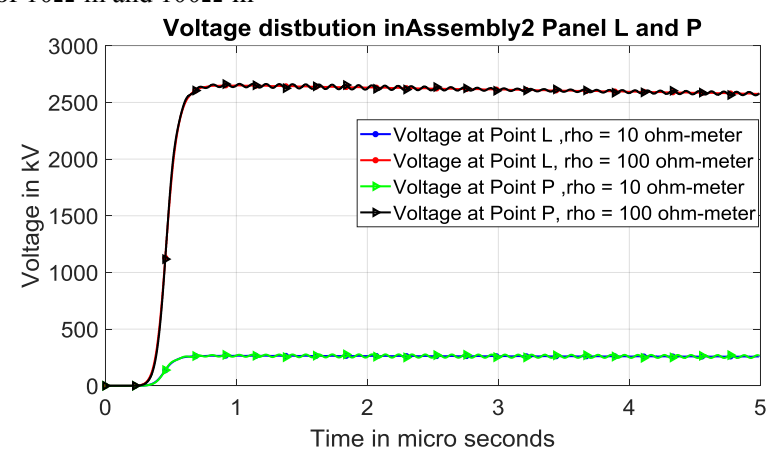

Figure 9 Potential drops in Assembly 2 - end-grounding

\section{B. Group Assembly-Middle-point ground}

Potential drops at locations A I L and P in case of middlepoint ground are shown in Figure 10 - Figure 13. Referring to Figure 10, voltage drop at the injection point has reached $2875 \mathrm{kV}$ for soil resistivity of $100 \Omega$-meter which is $40 \%$ more comparing the voltage drop at point $\mathrm{A}$ of the same PV system installed in a soil resistivity of $10 \Omega$-meter. It is evident that voltage drop at point I having soil resistivity $100 \Omega$-meter is $2656 \mathrm{kV}$ which $77 \%$ more compare to the voltage drop at $\mathrm{I}$ for the same system installed in a soil having soil resistivity of $10 \Omega$-meter.

Referring to Figure 12, 2644kV dropped at L in assembly 1 for soil having resistivity of $100 \Omega-\mathrm{m}$. This voltage drop is more than $83 \%(262 \mathrm{kV})$ of voltage dropped for the same system installed in soil having resistivity $10 \Omega$-meter. Voltage drop at point $\mathrm{P}$ of the assembly is of $2644 \mathrm{kV}$ which is $85 \%$ $(403 \mathrm{kV})$ more than that of voltage dropped in $\mathrm{P}$ of the same system installed in soil having resistivity of $10 \Omega$-meter.

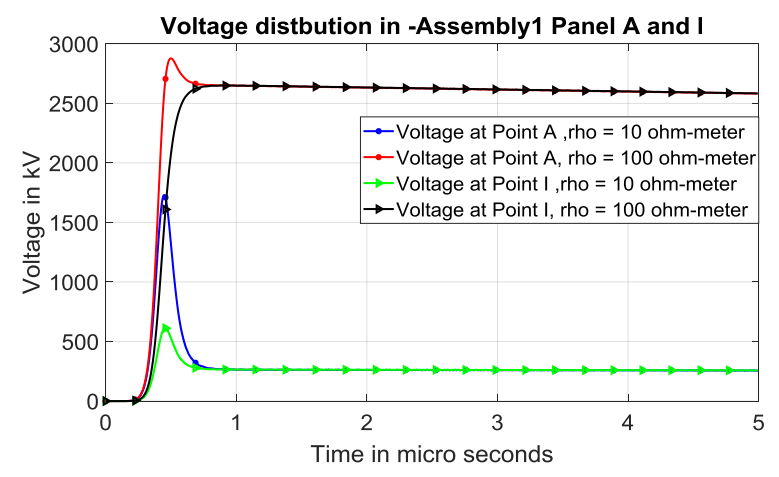

Figure 10 Potential drops in Assembly 1 - middle-grounding for soil resistivity of $10 \Omega-\mathrm{m}$ and $100 \Omega-\mathrm{m}$

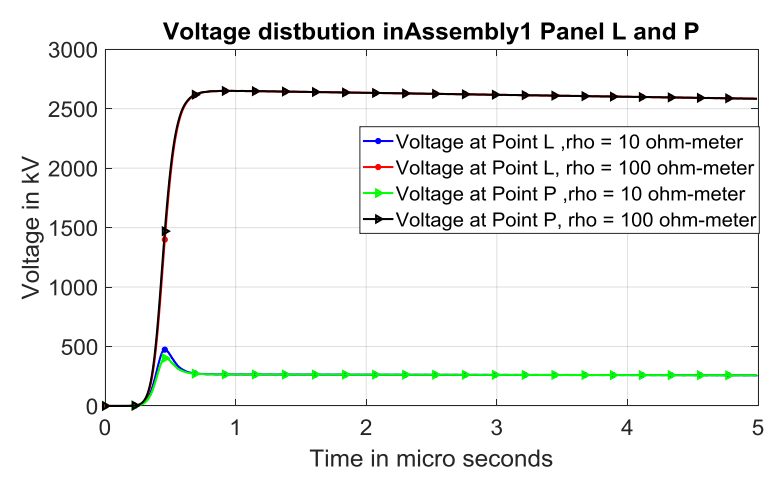

Figure 11 Potential drops in Assembly 1 - middle-grounding for soil resistivity of $10 \Omega-\mathrm{m}$ and $100 \Omega-\mathrm{m}$ 
Referring to Figure 12, Figure 13, Figure 14, Figure 15, Figure 16 and Figure 17, potential distribution in rest of the other assemblies (assemblies 2,3 and 4) are similar.

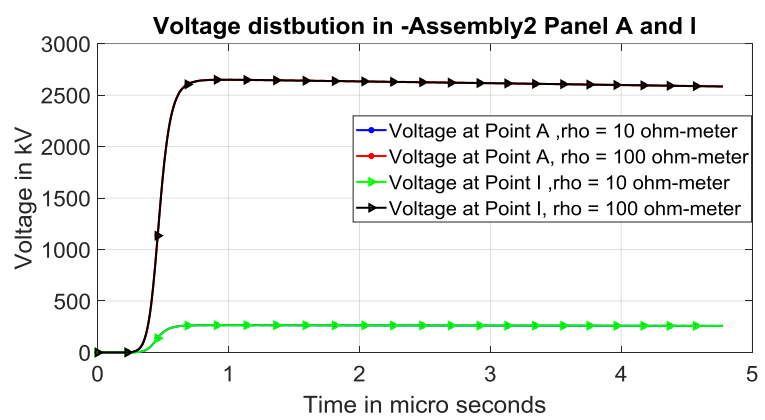

Figure 12 Potential drops in Assembly 2 - middle-grounding for soil resistivity of $10 \Omega-\mathrm{m}$ and $100 \Omega-\mathrm{m}$

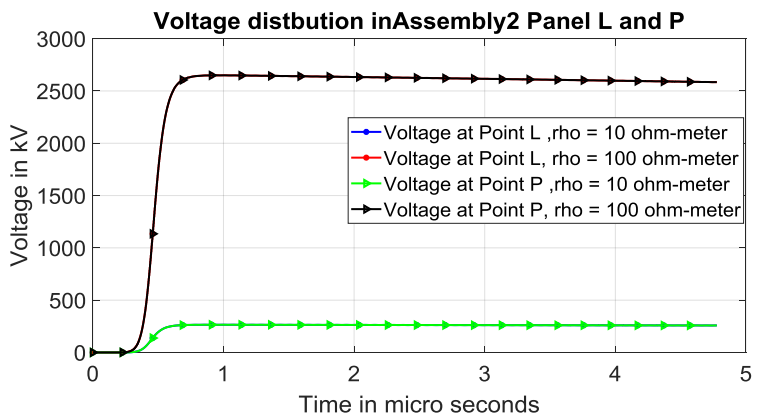

Figure 13 Potential drops in Assembly 2 - middle-grounding for soil resistivity of $10 \Omega-\mathrm{m}$ and $100 \Omega-\mathrm{m}$

For the soil resistivity of $10 \mathrm{ohm}$-meter, in group assemblies having end grounds potential drop at the point of lightning strokes is $30 \%$ higher than middle grounding. This offset increases to $62 \%$ at the point P. Potential drops across rest of the assemblies 2 to 4 shows high degree of similarity in both method of grounding. Hence voltage drop across the other points in the assembly in which lightning strikes, middle point grounding shows lower potential drops compare to end grounding.

\section{CONCLUSION AND FUTURE WORK}

Solar PV panels are exposed to lightning strikes which can affect function and life cycle of the panels. Effects of direct lightning strikes onto a solar PV assembly by considering the overvoltage resulting on the system due to various grounding arrangements. In particular, this paper is focused on group grounding of solar PV panels in which effect of middle-grounding or end-grounding points to down conductors for various soil resistivity is compared for distributed voltage drops across solar PV panels.

Based on the simulation results, it is found that solar PV panels consisting of group assemblies having 'middle ground' show lower voltage drops compare to 'end ground'. It is also evident that voltage drops in the panels that were not struck have a similar profile whether 'middle ground' or 'end grounding' is deployed. During the energy dissipation process through the grounding system, if the electric field exceeds a certain threshold (critical electric field), soil ionisation around the electrodes up to a certain radial distance may occur thus affecting the effective soil resistivity. Impact of soil ionization on the voltage drop due to lightning needs to be considered and will be considered in future work. Furthermore, authors are working on various options to conduct experimental analysis for the verification of the simulation results.

\section{REFERENCES}

[1] Benesova, Z., R. Haller, et al. (2012). Overvoltages in photovoltaic systems induced by lightning strikes. 2012 International Conference on Lightning Protection (ICLP).

[2] Fallah, N., C. Gomes, et al. (2013). Lightning protection techniques for roof-top PV systems. 2013 IEEE 7th International Power Engineering and Optimization Conference (PEOCO).

[3] Ayub, A. S., W. H. Siew, et al. (2018). Grounding strategies for solar PV panels. 2018 IEEE International Symposium on Electromagnetic Compatibility and 2018 IEEE Asia-Pacific Symposium on Electromagnetic Compatibility (EMC/APEMC).

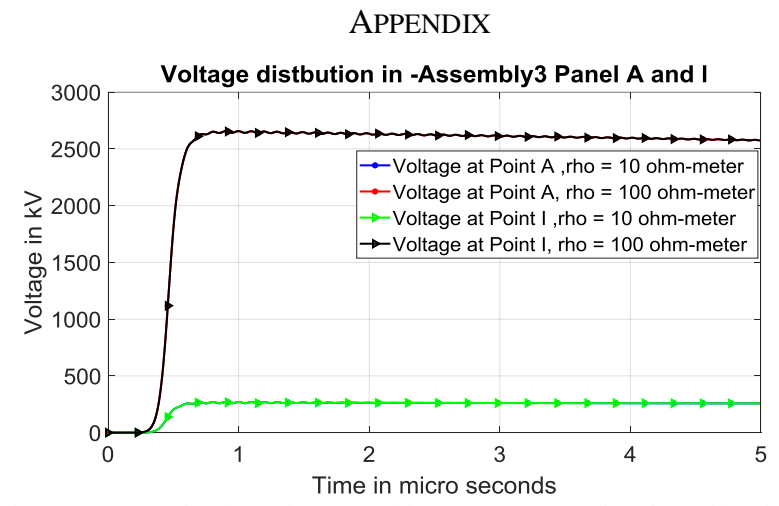

Figure 14 Potential drops in Assembly 3 - end-grounding for soil resistivity of $10 \Omega-\mathrm{m}$ and $100 \Omega-\mathrm{m}$

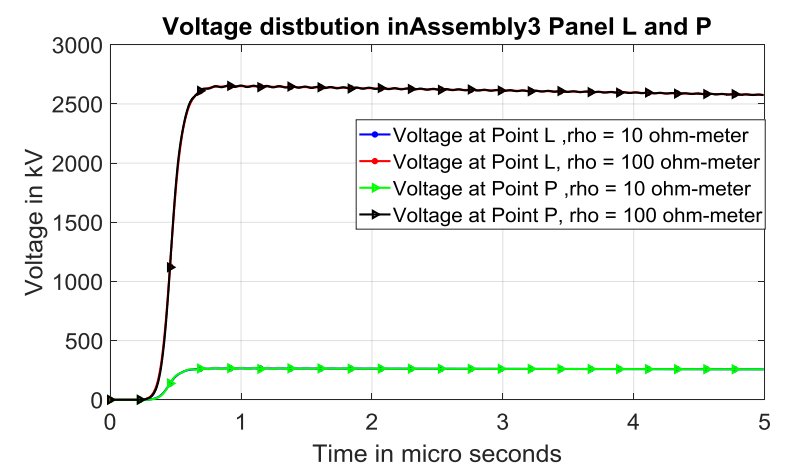

Figure 15 Potential drops in Assembly 3 - end-grounding for soil resistivity of $10 \Omega-m$ and $100 \Omega-m$

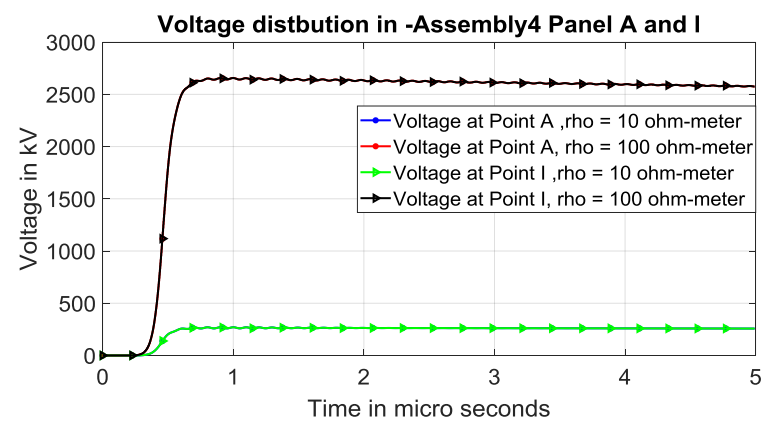

Figure 16 Potential drops in Assembly 3 - end-grounding for soil resistivity of $10 \Omega-\mathrm{m}$ and $100 \Omega-\mathrm{m}$ 


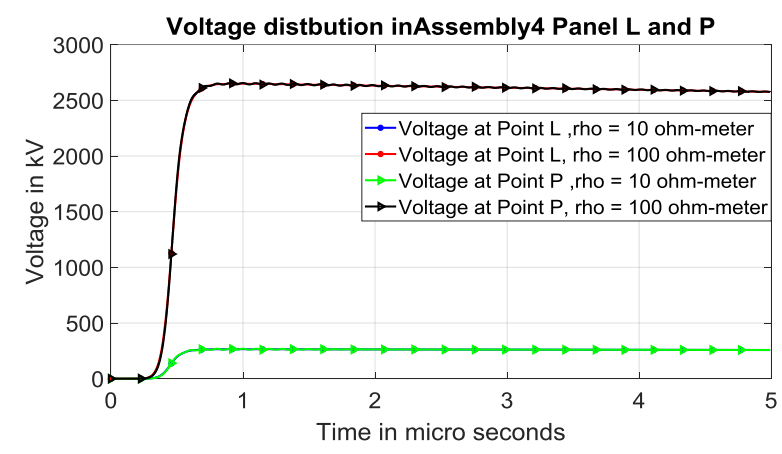

Figure 17 Potential drops in Assembly 4 - end-grounding for soil resistivity of $10 \Omega-\mathrm{m}$ and $100 \Omega-\mathrm{m}$

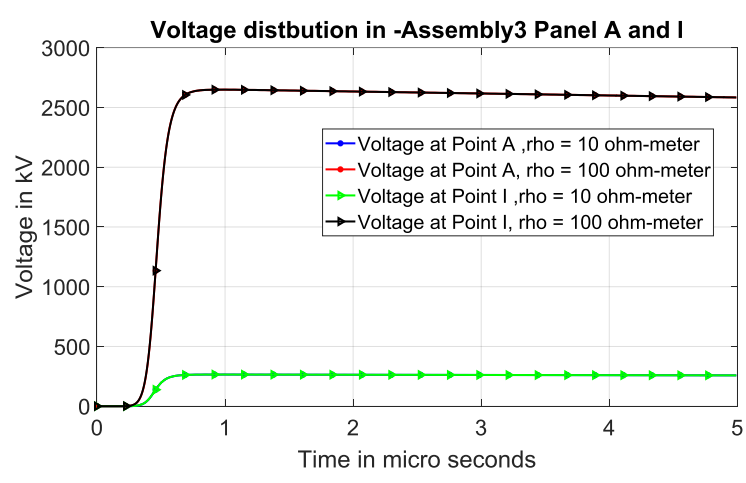

Figure 18 Potential drops in Assembly 3 - middle-grounding for soil resistivity of $10 \Omega-m$ and $100 \Omega-m$

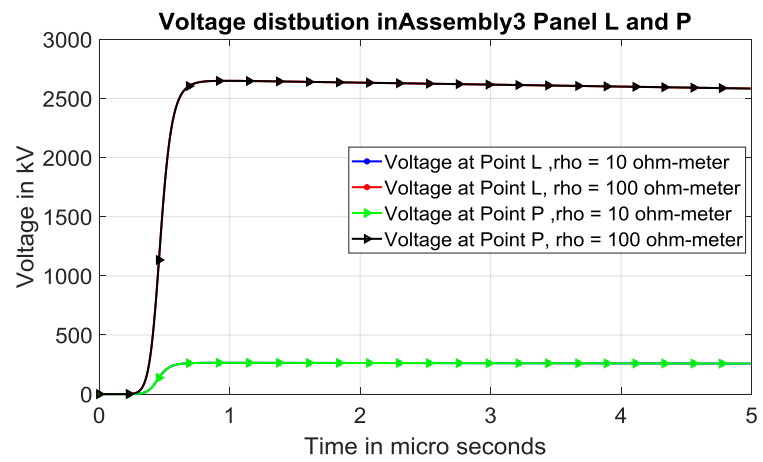

Figure 19 Potential drops in Assembly 3 - middle-grounding for soil resistivity of $10 \Omega-m$ and $100 \Omega-m$

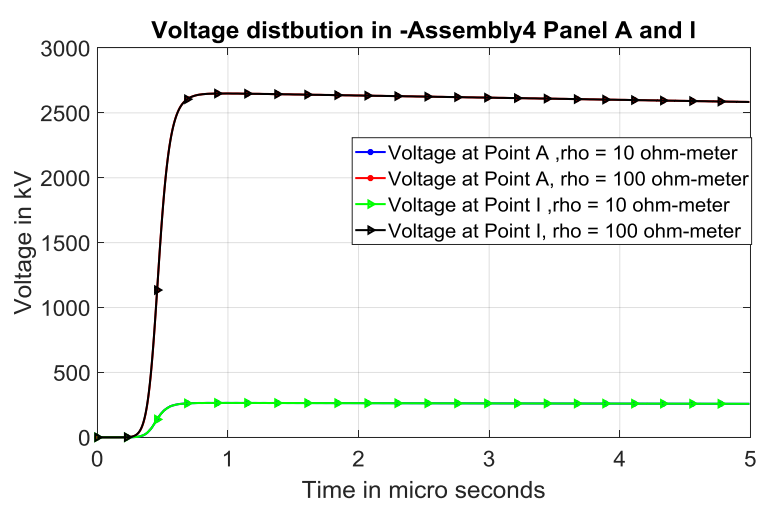

Figure 20 Potential drops in Assembly 4 - middle-grounding for soil resistivity of $10 \Omega-m$ and $100 \Omega-m$

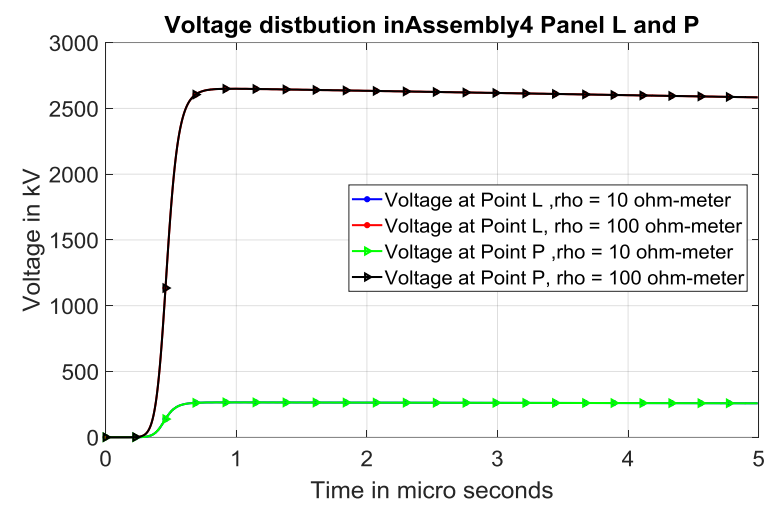

Figure 21 Potential drops in Assembly 4 - middle-grounding for soil resistivity of $10 \Omega-\mathrm{m}$ and $100 \Omega-\mathrm{m}$ 